\title{
ULTRASSONOGRAFIA DOPPLER APLICADA AO DIAGNÓSTICO DE DISTÚRBIOS TESTICULARES EM GARANHÕES
}

\author{
Deborah Freitas Silva ${ }^{1}$ \\ Gabriel Augusto Monteiro ${ }^{1}$
}

\begin{abstract}
RESUMO
As injúrias vasculares afetam diretamente a produção e a qualidade das células espermáticas, portanto, o diagnóstico rápido dessas alterações é de extrema importância para evitar danos irreversíveis à reprodução. Desse modo, a ultrassonografia Doppler têm se mostrado um método eficaz no diagnóstico precoce de afecções reprodutivas relacionadas com distúrbios na perfusão sanguínea testicular. Além disso, possibilita o acompanhamento de tratamentos em curso, a fim de melhorar resultados terapêuticos e proporcionar melhor previsão de fertilidade aos garanhões. Em homens, já é um método empregado para diagnosticar distúrbios de fertilidade, entretanto, na veterinária os relatos ainda são escassos e o uso por andrologistas de equinos para este fim, é esporádico. O objetivo dessa revisão é apresentar os princípios da ultrassonografia Doppler e os benefícios para a andrologia de equinos, afim de ampliar o conhecimento a respeito da técnica e facilitar o diagnóstico de afecções durante exames reprodutivos de garanhões.
\end{abstract}

Palavras-chaves: andrologia, artéria testicular, equinos, ultrassom e subfertilidade.

\section{DOPPLER ULTRASONOGRAPHY APPLIED TO THE DIAGNOSIS OF TESTICULAR DISORDERS IN STALLIONS}

\begin{abstract}
Vascular disturbance directly affects sperm production and quality, so the rapid diagnosis of these changes is extremely important to avoid irreversible damage to reproduction activity. Thereby, Doppler ultrasonography has been shown to be an effective method in the early diagnosis of reproductive disorders related to testicular blood perfusion. In addition, it's possible to monitor treatments in progress for the purpose of to improve therapeutic results and provide better prediction of fertility for stallions. In men, it is already a method used to diagnose fertility disorders, however, in the veterinarian routine the reports are still scarce and the use by equine andrologists for this purpose is sporadic. Thus, the aim of this review is to present the principles of Doppler ultrasonography and its benefits for the equine andrology, in order to expand knowledge about the technique and facilitate the diagnosis of diseases during reproductive examinations of stallions.
\end{abstract}

Keywords: andrology, testicular artery, equine, ultrasound and subfertility.

\section{ULTRASONOGRAFÍA DOPPLER APLICADA AL DIAGNÓSTICO DE DISTURBIOS TESTICULARES EN GARAÑONES}

\section{RESUMEN}

Las afecciones vasculares afectan directamente la producción y la calidad de las células

\footnotetext{
${ }^{1}$ Universidade Federal de Minas Gerais-Belo Horizonte, MG. Correspondência: deborah.fsilva@ @otmail.com.
}

Silva DF, Monteiro GA. Ultrassonografia doppler aplicada ao diagnóstico de distúrbios testiculares em garanhões. Vet. e Zootec. 2020.; 27: 001-017. 
espermáticas, por lo tanto, el diagnóstico temprano de estas alteraciones es de extrema importancia para evitar daños irreversibles en la reproducción. En este sentido, la ultrasonografía Doppler ha sido empleada como un método eficaz en el diagnóstico precoz de afecciones reproductivas relacionadas con disturbios en la perfusión sanguínea testícular. Además, este método posibilita el acompañamiento de tratamientos en curso, con el fin de mejorar resultados terapéuticos y obtener una predicción de la fertilidad de los garañones. En hombres, ya es um método empleado para el diagnóstico de disturbios de la fertilidad, mientras, en veterinaria los relatos aún son escasos y el uso por andrólogos en el campo equino para este fin, es esporádico. El objetivo de esta revisión es presentar los principios de la ultrasonografía Doppler y las ventajas para la andrología equina, con la finalidad de ampliar los conocimientos sobre esta técnica y facilitar el diagnóstico de afecciones durante los examenes reproductivos de los garañones.

Palabras claves: andrología, arteria testicular, equino, ultrasonido y subfertilidad.

\section{INTRODUÇÃO}

A ultrassonografia possibilita um diagnóstico não invasivo, rápido e é aplicada em estudos da anatomia, ecogenicidade, textura e mensuração do volume testicular (1), além do fluxo sanguíneo a partir da avaliação Doppler (2). A ultrassonografia Doppler surgiu com o objetivo de permitir exames mais detalhados de órgãos de diferentes sistemas e transmitir informações sobre a hemodinâmica dos vasos sanguíneos e sua arquitetura (3).

$\mathrm{Na}$ andrologia, o Doppler está sendo aplicado para avaliar a perfusão testicular (4), que está intimamente ligada com o bom funcionamento dos testículos e epidídimos, possibilitando o diagnóstico de suas patologias (5). Em homens, já é empregado para diagnosticar distúrbios de fertilidade (1), entretanto, nos garanhões os parâmetros geralmente utilizados para predizer disfunções reprodutivas são inespecíficos e tardios $(6,7)$, o que pode comprometer a fertilidade futura de animais importantes e valiosos para a reprodução.

A queda da produção espermática e qualidade seminal pode ser resultado de diversos fatores, desde processos agudos como traumas e aumento da temperatura do testículo, ou patologias primárias que levam à disfunção testicular, como a varicocele. Dessa forma, a identificação precoce da origem do distúrbio e o tratamento rápido evita a instalação de processos degenerativos e irreversíveis (6)

Nesse contexto, as modalidades ultrassonográficas surgiram como ferramentas capazes de amplificar a capacidade de diagnóstico e de monitoramento da resposta à tratamentos, por meio do status da perfusão testicular, com consequente melhoria do prognóstico à fertilidade dos garanhões $(7,8,9)$.

\section{ANATOMIA DO SISTEMA REPRODUTOR MASCULINO}

O aparelho reprodutor do garanhão é formado pelos testículos e epidídimos que são sustentados pelo cordão espermático e o músculo cremáster; também é formado pelo pênis, uretra associada, músculo isquiocavernoso, bulboesponjoso, retrator do pênis e pelas glândulas acessórias: glândulas vesiculares, próstata, bulbouretrais e ampolas (10).

O testículo é envolto por uma cápsula de tecido conjuntivo denso, a túnica albugínea, formada principalmente por fibras de colágeno (11). A túnica albugínea emite septos em direção ao parênquima testicular fornecendo sustentação e dividindo o órgão em lóbulos, onde são alojados os túbulos seminíferos. Os septos convergem medialmente formando o 
mediastino, região que nos equinos adultos só é visualizada microscopicamente, uma particularidade da espécie equina (12).

Os testículos situam-se fora da cavidade abdominal e são envolvidos por uma bolsa, o escroto, oriundo da pele e da fáscia que recobre o abdome. O escroto é dividido em quatro camadas: pele, túnica dartus, fáscia escrotal e túnica vaginal parietal (11).

A pele é a camada mais externa do escroto e é rica em glândulas sudoríparas que são componentes essenciais no mecanismo de termorregulação testicular. A camada de musculatura lisa adjacente à pele, a túnica dartus, também é responsável pelo controle da temperatura dos testículos por meio de sua contração ou relaxamento. A terceira camada que fica entre a túnica dartus e a túnica vaginal é a fáscia escrotal, composta de tecido conjuntivo frouxo que permite maior mobilidade do testículo no interior do escroto (13). Por fim, a túnica vaginal é uma camada dupla de mesotélio originado do peritônio, no qual o folheto parietal está adjacente ao escroto e o folheto visceral à túnica albugínea. Entre a túnica vaginal parietal e a túnica vaginal visceral há uma cavidade que contém um líquido seroso que serve como lubrificante e facilita o movimento do testículo (10).

O cordão espermático é constituído pelos ductos deferentes, vasos linfáticos, nervos, artéria e veia testicular, e o músculo cremáster (12). A artéria testicular se apresenta de forma altamente contorcida envolvida por uma rede de veias que drenam o testículo, chamada de plexo pampiniforme, o qual funciona como um mecanismo de troca de calor que reduz a temperatura do sangue que chega aos testículos $(10,14)$. O músculo cremáster se apresenta externamente ao cordão espermático, é formado a partir do músculo oblíquo abdominal interno e tem função de sustentação, além da termorregulação testicular (14).

O epidídimo por sua vez, é dividido anatomicamente em três segmentos, cabeça, corpo e cauda. Ao longo dos três segmentos há a diferenciação do epitélio, com redução da sua altura e dos estereocílios e aumento do lúmen (11). Funcionalmente, possui o segmento inicial, que corresponde à porção inicial da cabeça e o epitélio dos ductos eferentes e são responsáveis pela absorção de fluidos. O segmento médio, cabeça e corpo, realizam a maturação espermática; e o segmento final, que é composto pela cauda e porção dos ductos deferentes, executa o papel de armazenamento e manutenção de espermatozoides maduros $(14,15)$.

\section{Anatomia vascular}

A artéria testicular se origina da aorta abdominal e se alonga para acompanhar a migração do testículo para o escroto, apresentando-se altamente convoluta (16). Essa migração do testículo para a bolsa escrotal caracteriza a artéria marginal, com paredes mais finas e de menor diâmetro do que no cordão espermático (17).

Após o trajeto pelo cordão espermático, a artéria testicular apresenta um trajeto sinuoso sob a túnica albugínea, atingindo primariamente a borda epididimária cranial, segue pelo corpo do epidídimo no plano medial ou lateral no sentido da extremidade caudal do órgão, cede numerosos ramos mediais e laterais para a região mediastinal (18), e retorna ao plexo pampiniforme do cordão espermático para o abdome (19). A veia central por sua vez, tem início no centro da região caudal do testículo, segue cranio-dorsalmente e, após atravessar a túnica albugínea, emite ramificações que formam o plexo pampiniforme, sendo responsável por drenar o sangue dos testículos (20).

A imagem ultrassonográfica das artérias é anecóica, enquanto as veias possuem uma aparência mais hipoecóica. A veia central é encontrada na seção transversal como uma região circular hipoecóica e quando em seção longitudinal, é vista como uma linha ondulada. Já as 
veias do plexo pampiniforme têm diâmetros muito pequenos e não são facilmente identificadas (21).

\section{Termorregulação testicular}

As células espermáticas são sensíveis à ação do calor, desse modo, o testículo equino deve permanecer cerca de 3 a $4^{\circ} \mathrm{C}$ abaixo da temperatura corporal para obter um funcionamento eficaz (10). Portanto, características anatômicas são responsáveis por regular a temperatura intratesticular e evitar distúrbios na espermatogênese (11).

A termorregulação testicular ocorre a partir da associação de alguns mecanismos, tais como, a produção de secreção apócrina a partir da bolsa escrotal, o fluxo sanguíneo em contracorrente, ação da túnica dartus e do músculo cremáster (22).

A localização dos testículos fora da cavidade abdominal aumenta a superfície para dissipação de calor para o ambiente. Ademais, a pele delgada do escroto, com pêlos raros ou ausentes, pouca gordura subcutânea e grande quantidade de glândulas sudoríparas são mecanismos que facilitam a perda de temperatura $(22,23)$.

O músculo cremáster e a túnica dartus, que são regulados por nervos simpáticos da porção lombar, respondem às mudanças de temperatura ambiental afastando ou aproximando os testículos da região abdominal (22). Em dias com temperaturas mais baixas há a contração de ambas as musculaturas, o que reduz a troca de calor com o ambiente e mantém os testículos próximos do abdome. Já em dias com temperaturas elevadas ocorre o relaxamento das fibras musculares, com o intuito de afastar os testículos da região abdominal aquecida e permitir maior troca de calor (14).

O plexo pampiniforme proporciona uma superfície de contato e de troca de calor entre a artéria e veia testicular pelo mecanismo de contracorrente (22). Neste mecanismo, o sangue arterial que chega ao testículo é resfriado pelo sangue que retorna via plexo pampiniforme, orientando-o a uma temperatura média de $34^{\circ} \mathrm{C}$ a $35^{\circ} \mathrm{C}(10,11)$.

\section{ULTRASSONOGRAFIA DOS TESTÍCULOS E CORDÃO ESPERMÁTICO}

O exame ultrassonográfico é uma ferramenta eficaz e segura para avaliação da estrutura interna dos órgãos genitais, fornecendo diagnóstico precoce de diferentes patologias (24).

A sonda com frequência de $5-7,5 \mathrm{MHz}$ é ideal para avaliar a artéria testicular no cordão espermático $(2,4)$. No entanto, um transdutor com frequência mais alta (7,5-17 MHz) é mais adequado para o exame da artéria capsular e vasos do parênquima testicular (2). Para a realização do exame, o transdutor transretal linear é mais adequado, pois facilita o acesso aos testículos na região inguinal entre os membros posteriores.

\section{Modo B}

A ultrassonografia em escala cinza ou modo B fornece uma análise estrutural do órgão e é aplicada em estudos da anatomia, ecogenicidade, textura e mensuração do volume testicular (1), além de servir como plano de fundo para a representação colorida do fluxo sanguíneo a partir da avaliação Doppler (2).

Silva DF, Monteiro GA. Ultrassonografia doppler aplicada ao diagnóstico de distúrbios testiculares em garanhões. Vet. e Zootec. 2020; 27: 001-017. 


\section{Doppler}

A ultrassonografia Doppler é uma tecnologia emergente relativamente recente na medicina veterinária que propicia informações da vasculatura de vários órgãos, incluindo os testículos (25), logo, é uma técnica capaz de amplificar a capacidade de diagnóstico e de monitoramento no âmbito da andrologia equina (2).

\section{Histórico e fundamentos da ultrassonografia Doppler}

No ano de 1842, Christian Johann Doppler descreveu pela primeira vez a teoria do efeito Doppler, no qual ele observou que a frequência de uma onda sonora ou eletromagnética produzida por uma fonte que está em movimento se altera em relação ao receptor, aumentando com a aproximação e diminuindo com o distanciamento. A comprovação experimental ocorreu três anos mais tarde pelo meteorologista holandês Christoph H. D. Buys Ballot, que realizou um experimento no qual uma banda musical tocava em cima de uma locomotiva em movimento, enquanto um conjunto de observadores registravam as notas ouvidas de acordo com as diferentes velocidades de aproximação e de afastamento da locomotiva (26).

Na medicina veterinária o efeito Doppler é exemplificado por meio da avaliação dos vasos sanguíneos, onde uma hemácia em movimento dentro dos vasos reflete a onda sonora emitida pelo transdutor (27). A frequência emitida pelo aparelho é constante, já a frequência dos ecos refletidos varia conforme a movimentação das hemácias em relação ao transdutor. A diferença entre a frequência do som que foi emitido e a frequência que foi refletida é chamada deslocamento de frequência Doppler (fd) (28).

Se o fluxo sanguíneo estiver em direção ao transdutor, a frequência dos ecos que retornam será mais alta, o que resulta em uma fd positiva; já se o fluxo sanguíneo estiver em sentido contrário ao do transdutor, se tem uma frequência mais baixa do que a que foi transmitida, e uma fd negativa (FIGURA 1) (29). Quando a fonte de onda, no caso a hemácia, se move de forma perpendicular ao transdutor, não há deslocamento Doppler, dessa forma, não são detectados sinais Doppler colorido pelo equipamento (2).

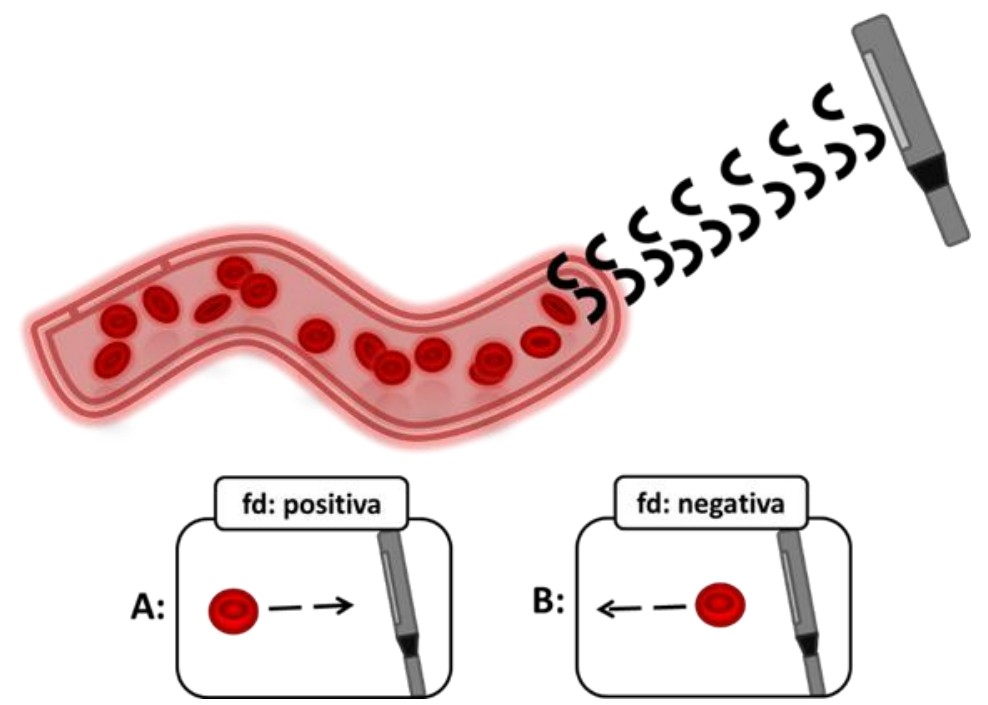

Figura 1. Ilustração representando a variação da frequência das ondas sonoras emitidas pelo transdutor e da frequência refletida. A: o fluxo sanguíneo com movimentação em direção ao transdutor reflete a onda com frequência maior que a emitida (fd positiva); B: o fluxo sanguíneo com movimentação no sentido oposto ao transdutor 
reflete a onda com frequência menor que a emitida (fd negativa). Fonte: Arquivo pessoal.

\section{MODALIDADES DOPPLER}

\section{Doppler colorido}

A avaliação do fluxo sanguíneo da artéria testicular pode ser realizada pelo Doppler colorido (30), que indica a velocidade, a presença e a direção do fluxo (28). O sinal recebido pelos elementos de amostragem do vaso é codificado por cores em relação ao sentido do fluxo e a velocidade do movimento (26). As diferentes cores indicam a direção do fluxo sanguíneo que também é demonstrada em uma barra colorida ao lado da imagem.

Convencionou-se que o fluxo que segue no sentido do transdutor é transmitido em vermelho e o fluxo que se afasta, em azul, porém, esse padrão pode ser manipulado pelo operador e, portanto, as cores podem ser variáveis. Já as intensidades das cores fornecem a ideia de velocidade, onde os fluxos de maior velocidade são expressos por tons mais claros da mesma cor (29).

\section{Power Doppler}

O Power Doppler é mais sensível que o Doppler colorido e, portanto, é utilizado para avaliar vasos de menor diâmetro e com menores velocidades de fluxo sanguíneo, especialmente no interior do parênquima testicular. Esse modo não detecta o sentido do fluxo e a velocidade, apenas expressa a presença ou não de fluxo sanguíneo no vaso avaliado. Devido a este fato, seu resultado independe do ângulo e não é influenciado por artefatos (2).

Essa modalidade detecta a amplitude ou potência do sinal Doppler e é muito eficiente no diagnóstico de ausência ou fluxo sanguíneo deficiente. A quantidade de células vermelhas transitando no vaso, ou seja, a intensidade do fluxo é representada como um gradiente de cor laranja sobreposto sobre a imagem do ultrassom modo B, que varia de laranja escuro para um laranja claro, representando menor e maior intensidade, respectivamente (21).

\section{Doppler pulsado ou espectral}

No modo espectral, o aparelho envia ondas curtas (pulsos) dentro de intervalos regulares (28) e a velocidade do fluxo sanguíneo é mensurada em função do tempo a partir da fd e do ângulo de insonação (30). O ângulo de insonação corresponde ao ângulo de intersecção do feixe do ultrassom com o fluxo sanguíneo (Figura 2), sendo que para obter informações mais confiáveis o ângulo deve estar entre $30^{\circ}$ e $60^{\circ}(2,4)$.

A fd e o ângulo Doppler são utilizados pelo instrumento para calcular a velocidade do sangue, como mencionado, a partir da fórmula:

$$
\mathbf{f d}=2 \mathrm{ft} \cdot \mathrm{V} \cdot \cos \theta / \mathrm{c}
$$

- $\mathrm{fd}=$ deslocamento de frequência Doppler

- $\mathrm{ft}=$ frequência do transdutor;

- $\mathrm{V}=$ velocidade das hemácias;

- $\theta=$ ângulo Doppler (ângulo formado pela intersecção do eixo correspondente à direção do fluxo sanguíneo dentro do vaso);

- $\cos \theta=\operatorname{coseno}$ do ângulo formado entre o transdutor e as hemácias; 
- $\mathrm{C}=$ velocidade média do ultrassom nos tecidos (fixada em 1540m.s-1).

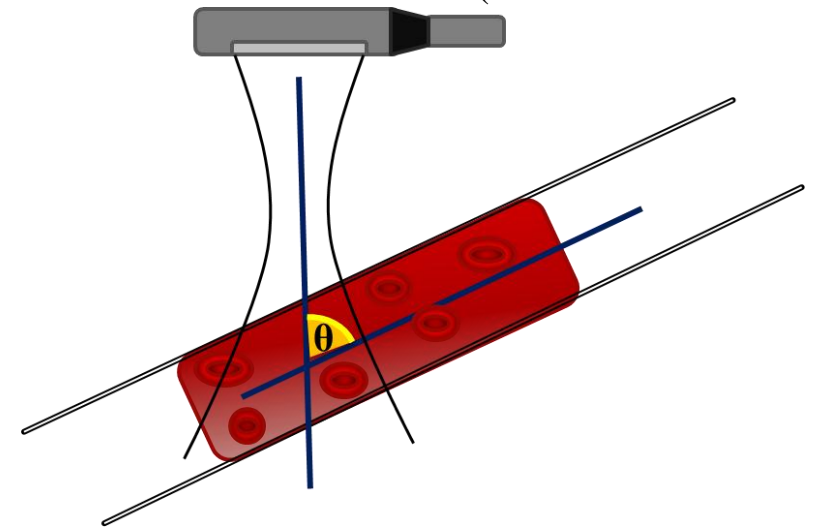

Figura 2. Esquema representando o ângulo formado entre o feixe proveniente do ultrassom com o fluxo sanguíneo do vaso avaliado: ângulo de insonação- $\theta$ (marcação em amarelo na figura). Fonte: Arquivo pessoal.

Ao ativar o modo espectral, os resultados do campo de amostragem do vaso avaliado são expressos em um gráfico denominado espectro, que representa a mudança de velocidade ao longo do tempo em associação com os pulsos dos ciclos cardíacos (21). Convencionou-se que o traçado espectral se dispõe acima do zero da linha base (desvio positivo) nos casos em que a frequência retornada for maior que a frequência emitida e o fluxo estiver na mesma direção do transdutor. Já quando a frequência retornada for menor do que a que foi emitida pelo aparelho e o fluxo estiver no sentido contrário ao transdutor, o traçado se dispõe abaixo da linha base (desvio negativo) (25).

O espectro fornece os valores de velocidade do pico sistólico (VPS), velocidade diastólica final (VDF) e velocidade máxima média (TAMV) (21). O VPS corresponde ao valor máximo no decorrer do espectro, VDF representa a velocidade final de um ciclo cardíaco e o TAMV é a média dos valores máximos durante todo gráfico (Figura 3) (2).

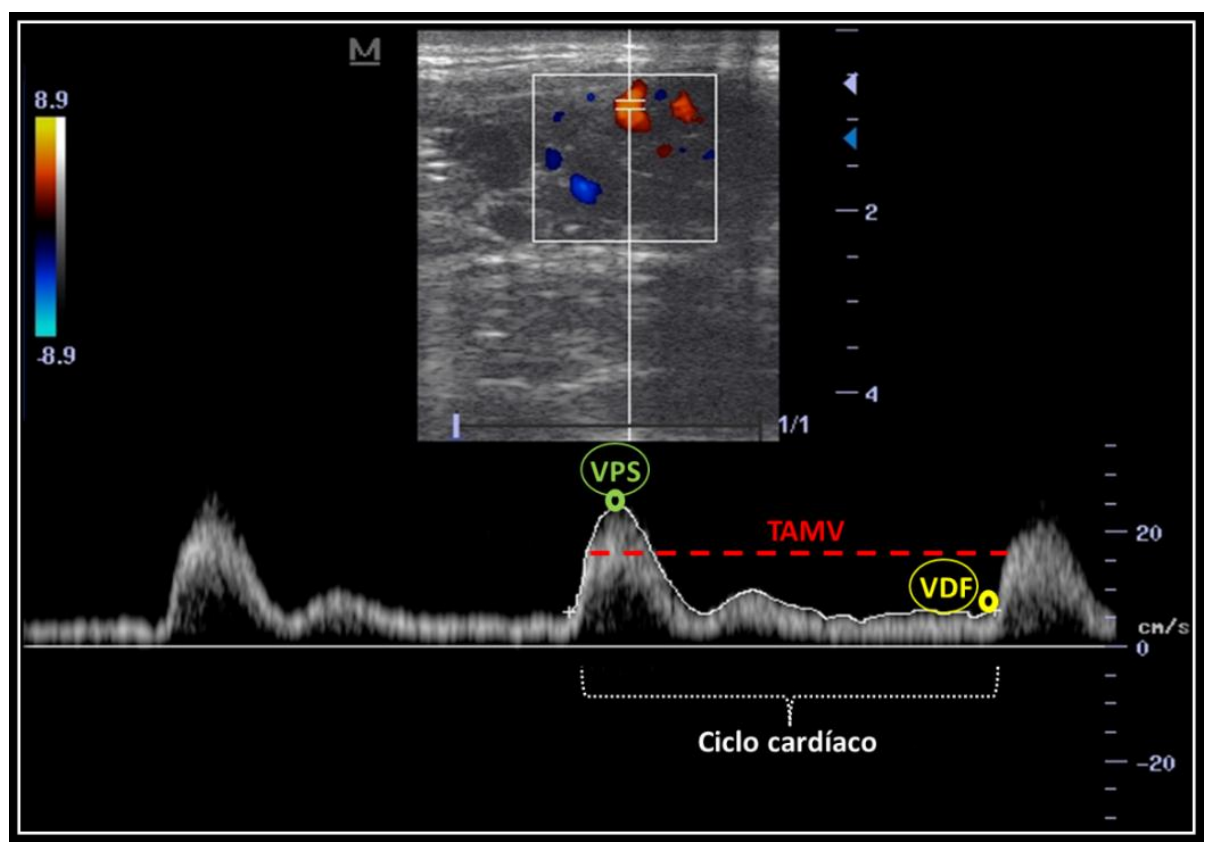

Figura 3. Espectro demonstrando a velocidade do fluxo sanguíneo ao longo de um ciclo cardíaco, com destaque para os valores de velocidade do pico sistólico (VPS), velocidade diastólica final (VDF) e velocidade máxima média (TAMV). Fonte: Arquivo pessoal.

Silva DF, Monteiro GA. Ultrassonografia doppler aplicada ao diagnóstico de distúrbios testiculares em garanhões. Vet. e Zootec. 2020.; 27: 001-017. 
Como a impedância vascular não pode ser obtida apenas pelo valor de velocidade absoluta, os índices Doppler, índice de resistência (IR) e índice de pulsatilidade (IP), foram desenvolvidos. Esses índices são razões calculadas a partir de vários pontos do espectro por meio das três velocidades já citadas: $\mathrm{IR}=(\mathrm{VPS}-\mathrm{VDF} / \mathrm{VPS})$ e IP=(VPS-VDF/TAMV). O IR indica a resistência ao fluxo sanguíneo que pode ser gerada por aumento da viscosidade sanguínea, redução do diâmetro do vaso ou por vasos muito compridos. Já o IP indica a pulsatilidade da forma de onda $(2,31)$.

Os índices Doppler independem do ângulo de insonação e, portanto, são utilizados em artérias pouco calibrosas ou muito tortuosas, no qual fica mais difícil estimar o ângulo. $\mathrm{O}$ aumento de IR e IP aponta redução da perfusão ao tecido ou órgão, sendo o IR mais sensível e por isso já vêm sendo utilizado no diagnóstico de transtornos do fluxo sanguíneo testicular em humanos (32).

Um fluxo sanguíneo com alto IR e IP gera um gráfico com picos sistólicos mais afilados e fluxo reverso no início da diástole. Já um padrão de fluxo de baixa resistividade e pulsatilidade são caracterizados por picos sistólicos amplos e contínuos e alta velocidade de fluxo na diástole, sendo a velocidade decrescente $(28,29)$.

Outros parâmetros de avaliação da perfusão sanguínea foram introduzidos por apresentarem resultados mais satisfatórios e mais precoces: o fluxo sanguíneo arterial total (TABF) e a taxa de fluxo sanguíneo arterial total (Tx TABF), calculados pelas fórmulas a seguir (33):

\section{TABF: TAMV $x$ A}

- $\mathrm{TABF}=$ fluxo sanguíneo arterial total;

- TAMV= velocidade máxima média;

- $\mathrm{A}=$ área da seção transversal do vaso $(\mathrm{A}=\pi \mathrm{r} 2)$;

- $\mathrm{r}=$ diâmetro arterial/2;

- $\pi=3,14$

- (unidade de medida: $\mathrm{ml} / \mathrm{min}$ ).

Taxa TABF: TABF/ VTT $x 100$

- Taxa $\mathrm{TABF}=$ taxa de fluxo sanguíneo arterial total;

- TABF= fluxo sanguíneo arterial total;

- VTT= volume testicular total;

- (unidade $\mathrm{ml} / \mathrm{min} / 100 \mathrm{~g}$ ).

Nos garanhões, a artéria testicular é geralmente avaliada no cordão espermático (artéria supratesticular), e/ou no pólo caudal do testículo (artéria capsular ou marginal) e/ou no parênquima testicular (artéria intratesticular) (FIGURA 5). Entretanto, é mais facilmente visualizada na região do cordão quando comparado ao ramo marginal, em função do maior calibre (4). 


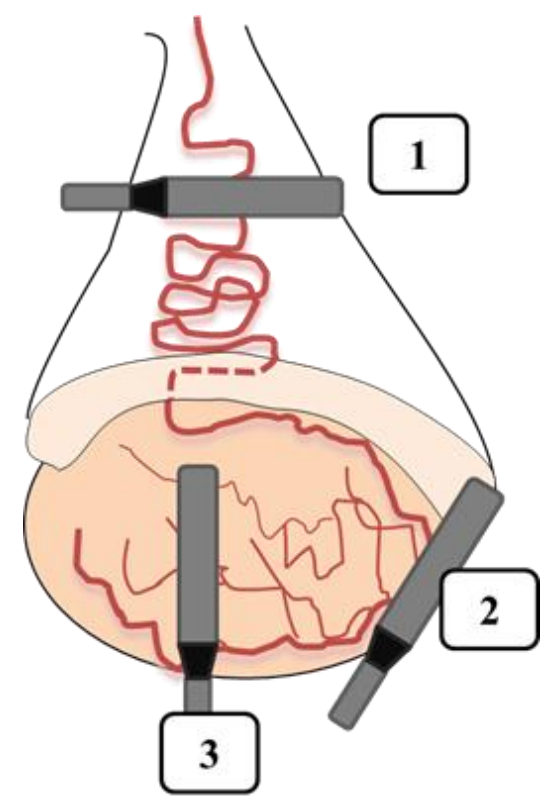

Figura 4. Esquema da artéria testicular com ênfase nas três regiões de avaliação com o Doppler espectral e orientação do transdutor. 1) Cordão espermático: artéria supratesticular; 2) Próximo à cauda do epidídimo: artéria capsular ou marginal e, 3) dentro do parênquima: artéria intratesticular. Fonte: Arquivo pessoal.

\section{PARÂMETROS DOPPLER NA AVALIAÇÃO DA FUNÇÃO TESTICULAR}

Os equinos são normalmente selecionados para criação com base em sua linhagem, capacidade atlética e outras características fenotípicas. Desse modo, o potencial reprodutivo é geralmente deixado em segundo plano e a seleção para uma boa fertilidade não é tão rigorosa quanto em outras espécies, como é o caso dos bovinos (34). Portanto, os garanhões geralmente apresentam índices de fertilidade inferiores em relação às demais espécies domésticas (35).

O suprimento sanguíneo adequado para os testículos é determinante para sua função, e de fato, as injúrias vasculares são uma das causas mais comuns de subfertilidade, podendo afetar o volume testicular e a produção espermática $(7,9)$.

A identificação precoce dessas falhas na perfusão sanguínea para os testículos é essencial para o diagnóstico de distúrbios testiculares, e para monitoramento dos resultados terapêuticos (9). Diante desse fato, alguns estudos foram realizados em diferentes espécies a fim de avaliar a relação dos índices Doppler (IR e IP) com os parâmetros relativos à funcionalidade testicular $(8,36,37)$.

No estudo de Zelli et al. (37) os resultados apontaram os índices Doppler como potenciais marcadores da qualidade seminal em cães, com correlação negativa entre IR e IP com os parâmetros de motilidade total (MT), motilidade progressiva (MP) e integridade de membrana. Nos garanhões, as correlações também foram negativas entre IR e IP com volume testicular, número total de espermatozoides e o número total de espermatozoides morfologicamente normais com MP $(7,8,9)$, e espermatozoides com alta atividade mitocondrial após 24 e $48 \mathrm{hrs} \mathrm{a} 5^{\circ} \mathrm{C}$ (7). Além disso, os índices Doppler foram positivamente correlacionados com o nível de FSH no sangue (8)

Altas concentrações plasmáticas de FSH são observadas em garanhões com subfertilidade ou infertilidade idiopática, decorrente da disfunção das células de Sertoli, com consequente falha no processo de feedback regulatório do FSH (38). Desse modo, o ensaio de FSH é muito utilizado para determinar disfunção testicular nessa espécie, entretanto, as

Silva DF, Monteiro GA. Ultrassonografia doppler aplicada ao diagnóstico de distúrbios testiculares em garanhões. Vet. e Zootec. 2020.; 27: 001-017. 
avaliações da função testicular a partir do Doppler espectral por meio dos resultados de IR e IP têm a vantagem de proporcionar diagnóstico mais rápido e prático (8).

\section{PARÂMETROS DOPPLER NA AVALIAÇÃO DE AFECÇÕES REPRODUTIVAS}

Vários fatores podem comprometer a função reprodutiva dos garanhões, desde afecções indiretas que impeçam a monta, como desordens no sistema locomotor, até disfunções que comprometam diretamente a qualidade seminal (39), como as desordens vasculares.

A modalidade Doppler espectral têm se mostrado uma técnica efetiva para complementar o diagnóstico de afecções reprodutivas e caracteriza uma forma alternativa aos métodos de rotina mais lentos e laboriosos (8). Nesse contexto, além de bons marcadores da espermatogênese (32), os índices Doppler foram considerados indicadores confiáveis para identificar distúrbios reprodutivos (36). Pinggera et al. (36) relataram que homens oligospérmicos apresentaram IR mais elevado ( $I R$ médio $=0,7)$ quando comparado a homens com contagem normal de espermatozoides (IR médio $=0,5$ ), no qual sugeriram que o IR superior a 0,6 implica em contagem patológica de espermatozoides.

Ademais, o IR juntamente com o VPS são úteis para diferenciar azoospermia obstrutiva da não obstrutiva em homens, com valores inferiores nos casos de azoospermia não obstrutiva. Porém, como as velocidades VPS e VDF dependem do ângulo de insonação para serem calculadas e, portanto, exige uma avaliação mais cuidadosa, o IR surge como alternativa rápida e eficaz $(32,36)$.

O estudo de Ortiz-Rodriguez e colaboradores (7) descreveu a redução no fluxo sanguíneo testicular dos garanhões subférteis, com redução significativa no diâmetro da artéria capsular $(0,32 \mathrm{~mm}$ vs $0,25 \mathrm{~mm})$. No qual, esses garanhões subférteis apresentaram valores inferiores de todas as velocidades Doppler (VPS, VDF, TAMV), e de TABF e Tx TABF, o que caracteriza uma menor perfusão sanguínea.

Geralmente os parâmetros utilizados para predizer disfunções reprodutivas são a eficiência espermática (DSOr (real) / DSOe (esperada)) e o volume testicular (VT: 0,53 x altura $\mathrm{x}$ comprimento $\mathrm{x}$ largura $\left(\mathrm{cm}^{3}\right)$ ), no qual o termo DSO corresponde ao número de espermatozoides que um garanhão pode produzir diariamente. De fato, foram encontradas diferenças significativas no VT, DSOr e DSOe entre grupos férteis e subférteis (7). Entretanto, esses parâmetros são indicadores inespecíficos e tardios, e quando a baixa eficiência espermática é notada, juntamente com alta porcentagem de espermatozoides anormais e redução do tamanho testicular, geralmente o dano ao testículo é relevante, com processos degenerativos já instalados $(6,7)$.

Desse modo, o Doppler espectral representa uma possibilidade de diagnóstico precoce das patologias reprodutivas associadas com alteração do fluxo sanguíneo, como torção do cordão espermático, hidrocele, varicocele, alterações inflamatórias testiculares/escrotais e neoplasias, possibilitando a intervenção com tratamento mais adequado e com melhores prognósticos (7).

\section{Torção do cordão espermático}

Anatomicamente, como já descrito, os testículos são sustentados pelo cordão espermático e possuem certa mobilidade dentro da bolsa escrotal. No entanto, uma mobilidade excessiva do testículo pode levar a uma rotação deste em torno do seu eixo vertical, de modo a estrangular o cordão, o que reduz ou cessa a perfusão sanguínea ao órgão (40).

Silva DF, Monteiro GA. Ultrassonografia doppler aplicada ao diagnóstico de distúrbios testiculares em garanhões. Vet. e Zootec. 2020.; 27: 001-017. 
O aparecimento de sinais clínicos vai depender do grau da torção e do tempo que está instalada. Se o retorno venoso for parcialmente obstruído haverá congestão e edema, em especial do epidídimo. Porém, em casos de obstrução total do retorno venoso a congestão é mais grave podendo ocorrer necrose rapidamente. Em casos de impedimento total do fluxo arterial, há infarto testicular por falta de oxigênio (41). Torções graves podem causar quadros de hidrocele e hematocele, com consequente degeneração testicular (42).

Torções inferiores a $180^{\circ}$ geralmente não causam sinais clínicos evidentes. O fluxo arterial ainda está presente, mas o fluxo venoso é interrompido e a dilatação venosa pode ser observada (9). Torções de $360^{\circ}$ são acompanhadas de dor aguda, semelhante à cólica e aumento do testículo e cordão afetados, representando normalmente uma emergência cirúrgica (43).

Pozor e McDonnell (4) relataram fluxo sanguíneo diastólico retrógrado em garanhões com torção do cordão de $180^{\circ}$ e sugeriram que apesar da ausência de sinais clínicos evidentes, essa afecção afeta o fluxo sanguíneo testicular. No entanto, são necessários mais estudos. Em humanos com torção incompleta também foi observado fluxo sanguíneo diastólico retrógado e aumento do IR (44).

\section{Hidrocele}

A hidrocele é o acúmulo não fisiológico de fluido seroso entre os folhetos da túnica vaginal. Pode ser do tipo comunicante, quando o processo vaginal está patente e permite a comunicação do líquido peritoneal com o escroto. Portanto, há fluxo de líquido em ambas as direções e o acúmulo de fluido é variável, aumentanto ou diminuindo de acordo com o grau de esforço ou repouso. E não comunicante, quando ocorre apenas a nível de escroto e o fluido surge do revestimento mesotelial da túnica vaginal. Nesse caso, o canal está fechado mas o fluido não foi reabsorvido, é o tipo mais comum e geralmente de resolução espontânea $(45,46)$.

A hidrocele pode ser observada em garanhões estabulados por longos períodos ou em garanhões com atividade sexual intensa após a estação reprodutiva (9). Alguns casos podem ser consequências de patologias primárias, como orquite, epididimite, neoplasias testiculares, torção do cordão espermático e traumas (43).

No estudo de Pozor e McDonnel (4) casos leves e moderados de hidrocele não afetaram a perfusão sanguínea testicular. No entanto, em casos graves com quantidade considerável de fluido circundando o testículo e cordão espermático foi evidente um fluxo sanguíneo turbulento próximo ao cordão. Em humanos foi reportado aumento de IR e IP nos casos de hidrocele leve (47), já em garanhões são escassos os estudos da relação dessa afecção com os parâmetros doppler.

\section{Varicocele}

A varicocele consiste na dilatação e tortuosidade das veias que percorrem o cordão espermático (48). O mecanismo de formação da varicocele ainda é pouco conhecido, entretanto, sua origem pode estar relacionada com a incompetência das válvulas que leva ao refluxo venoso e consequente acúmulo de substâncias tóxicas e com aumento da pressão hidrostática ao nível do plexo pampiniforme. Dessa forma, numa tentativa de manter a homeostasia da pressão intratesticular, o sangue oxigenado fica estacionado no sistema de microcirculação arterial e a chegada aos testículos é dificultada $(49,50)$.

Silva DF, Monteiro GA. Ultrassonografia doppler aplicada ao diagnóstico de distúrbios testiculares em garanhões. Vet. e Zootec. 2020; 27: 001-017. 
Nos humanos a varicocele é uma das patologias mais frequentemente associada com subfertilidade, visto que o estado de hipóxia e aumento de temperatura dos testículos afeta diretamente as células espermáticas em desenvolvimento, células de Sertoli e de Leydig $(51,52)$.

Humanos com varicocele assintomática a princípio parecem não ter alteração do fluxo sanguíneo e dos parâmetros Doppler, porém, homens com sinais clínicos apresentaram valores inferiores de TAMV em relação aos saudáveis (33). Nos garanhões também foi reportado uma alteração dos índices Doppler e da velocidade, com uma tendência à maiores valores de VPS, IR e IP na artéria supratesticular (4).

\section{Orquite e epididimite}

A orquite e epididimite correspondem a inflamações dos testículos e epididímos, respectivamente, que podem ter diferentes causas (53). Normalmente, a orquite está associada com a epididimite e nos equinos podem ser consequência de traumas, feridas perfurantes no testículo, migração de parasitas, agentes infecciosos por disseminação hematogênica e doença autoimune (54).

Em função da inflamação é observado pelo Color Doppler o aumento da perfusão sanguínea para o órgão afetado (hipervascularização). Estudos em humanos mostraram menor IR e maior VPS na artéria testicular de homens com orquite-epididimite em comparação com homens normais (55).

Entretanto, alguns estudos descreveram aumento do IR e fluxo diastólico retrógrado em pacientes com infarto e necrose testicular, resultante da complicação da orquite-epididimite (56). Nos garanhões, Ortega-Ferrusola et al. (9) também descreveram aumento de IR e fluxo diástólico retrógrado em um garanhão com suspeita de orquite-epididimite crônica que apresentava regiões hipoecóicas multifocais no parênquima de ambos os testículos, compatíveis com granuloma ou fibrose espermática.

\section{Neoplasia testicular}

As neoplasias testiculares podem ser classificadas em primárias e secundárias, sendo as primárias subdivididas em germinativas e não-germinativas, hormonalmente ativas ou não. As neoplasias germinativas têm origem de células germinativas do epitélio seminífero e incluem os seminomas, teratomas, teratocarcinomas e carcinoma embrionário. Os não-germinativos originam-se do estroma testicular e englobam os tumores das células de Leydig, tumores das células de Sertoli, lipomas e mastocitomas (57). As neoplasias secundárias do testículo são mais raras que as primárias (58).

A ultrassonografia em escala cinza em associação com o Doppler é uma ferramenta essencial para diferenciar estruturas sólidas localizadas nos testículos das paratesticulares, assim como, diferenciar massas neoplásicas das lesões císticas (59). Em um estudo com cães foi relatado sinais vasculares intensos ao redor e no interior das massas neoplásicas testiculares a partir da avaliação com o color Doppler, e nenhum fluxo foi detectado em estruturas císticas (60). Portanto, apesar do método de escolha para o diagnóstico de neoplasias testiculares ser o exame histopatológico, a ultrassonografia Doppler pode aumentar a confiabilidade do diagnóstico e evitar erros (60).

\section{CONSIDERAÇÕES FINAIS}


A ultrassonografia Doppler é um método promissor no auxílio ao diagnóstico precoce de disfunção testicular em garanhões e de grande potencial no monitoramento de resultados terapêuticos pós-tratamentos. Ademais, pode ser uma alternativa aos métodos diagnósticos tradicionais laboriosos e mais lentos que as modalidades Doppler.

\section{REFERÊNCIAS}

1. Schurich M, Aigner F, Frauscher F, Pallwein L. The role of ultrasound in assessment of male fertility. Eur J Obstet Gynecol Reprod Biol .2009;144:192-18.

2. Ginther OJ, Utt MD. Doppler ultrasound in equine reproduction: principles, techniques, and potential. J Equine Vet Sci. 2004;24:516-26.

3. Carvalho CF, Chammas MC, Cerri GG. Princípios físicos do Doppler em ultrassonografia. Cienc Rural. 2008;38:872-9.

4. Pozor MA, McDonnell SM. Color Doppler ultrasound evaluation of testicular blood flow in stallions. Theriogenology. 2004;61:799-810.

5. Guenzel-Apel AR, Moehrke C, Nautrup CP. Colour-coded and pulsed Doppler sonogragraphy of the canine testis, epididymis and prostate gland: physiological and pathological findings. Reprod Domest Anim. 2001;36:236-40.

6. Turner RM. Pathogenesis, diagnosis, and management of testicular degeneration in stallions. Clin Tech Equine Pract. 2007;6:278-84.

7. Ortiz-Rodriguez JM, Anel-Lopez L, Martín-Muñoz P, Alvarez M, Gaitskell-Phillips G, Anel L, et al. Pulse Doppler ultrasound as a tool for the diagnosis of chronic testicular dysfunction in stallions. Plos One. 2017;12:e017587.

8. Pozor MA, Nolin M, Roser J, Runyon S, Macperson ML, Kelleman A. Doppler index of vascular impedance as indicator of testicular dysfunction in stallions. J Equine Vet Sci. 2014;34:38-9.

9. Ortega-Ferrusola C, Gracia-Calvo LA, Ezquerra J, Pena FJ. Use of colour and spectral Doppler ultrasonography in stallion andrology. Reprod Domest Anim. 2014;49:88-96.

10. Amann RP. A review of anatomy and physiology of the stallion. J Equine Vet Sci. 1981;1:83-105.

11. Hafez ESE. Anatomia da reprodução masculina. In: Hafez B, Hafez ESE. Reprodução animal. 7a ed. Barueri: Manole; 2004. p.97-140.

12. Chenier ST. Anatomy and examination of the normal testicle. In: Pycock S, Samper JC, McKinnon OA. Current therapy in equine reproduction e-book. St. Louis: Saunders; 2007. cap.26, p.167-73.

13. Stickle RL, Fessler JF. Retrospective study of 350 cases of equine cryptorchidism. J Am Vet Med Assoc. 1978;172:343-6.

Silva DF, Monteiro GA. Ultrassonografia doppler aplicada ao diagnóstico de distúrbios testiculares em garanhões. Vet. e Zootec. 2020.; 27: 001-017. 
14. Amann RP. Functional anatomy of the adult male. In: McKinnon AO, Squires EL, Vaala E, Varner DD. Equine reproduction. 2nd ed. Philadelphia: Wiley-Blackwell; 2011. p.86780 .

15. Marengo SR. Maturing the sperm: unique mechanisms for modifying integral proteins in the sperm plasma membrane. Anim Reprod Sci. 2008;105:52-63.

16. Setchell BP. Testicular blood supply, lymphatic drainage and secretion of fluid. In: Johnson AD, Gomes WR, Vandemark NL. The testis. New York: Academic Press; 1970. v.1, p. 101-239.

17. Budras KD, Mccarthy PH, Fricke W, Richter R. Anatomy of the dog. London: Manson; 2007.

18. Pozor M, Kolonko D. The testicular artery of stallions in clinical and morphological studies. Med Weter. 2001;57:822-6.

19. Turner RM. Ultrasonography of the genital tract of the stallion. In: Reef VB. Equine diagnostic ultrasound. Philadelphia: Wiley-Blackwell; 1998. p.446-79.

20. Love CC. Ultrasonographic evaluation of the testis, epididymis, and spermatic cord of the stallion. Vet Clin North Am Equine Pract. 1992;1:167-82.

21. Pozor MA. Evaluation of testicular vasculature in stallions. Clin Tech Equine Pract. 2007;6:271-7.

22. Gabaldi SH, Wolf A. A importância da termorregulação testicular na qualidade do sêmen em touros. Cienc Agrar Saude. 2002;2:66-70.

23. Setchell B, Maddocks S, Brooks D. Anatomy, vasculature, innervation and fluids of the male reproductive tract. In: Knobil E, Neill JD. The physiology of reproduction. New York: Raven Press; 1994.

24. Palmer E, Driancourt MA. Use of ultrasonic echography in equine gynecology. Theriogenology. 1980;13:203-16.

25. Carvalho CF, Chammas MC, Cerri GG. Princípios físicos do Doppler em ultrassonografia. Cienc Rural. 2008;38:872-9.

26. Kawakama J. Física. In: Cerri GG, Rocha DC. Ultra-sonografia abdominal. São Paulo: Sarvier; 1993. cap.1, p.1-14.

27. Vermillon RP. Basic physical principles. In: Snider AR, Serwer GA, Gersony RA. Echocardiography in pediatric heart disease. 2nd ed. Missouri: Mosby; 1997. cap.1, p.110.

28. Szatmári V, Sótonyi P, Voros K. Normal duplex Doppler waveforms of major abdominal blood vessels in dogs: a review. Vet Radiol Ultrasound. 2001;42:93-107. 
29. Cerri GG, Mólnar LJ, Paranaguá-Vezozzo DC. Doppler. São Paulo: Sarvier; 1998. Avaliação dúplex do fígado, sistema portal e vasos viscerais; p.120-1.

30. Dogra VS, Gottlieb RH, Oka M, Rubens DJ. Sonography of the scrotum. Radiology. 2003;227:18-36.

31. Wood MM, Romine LE, Lee YK, Richman KM, O’Boyle MK, Paz DA, et al. Spectral Doppler signatures waveforms in ultrasonography. Ultrasound Q. 2010;26:283-99.

32. Biagiotti G, Cavallini G, Modenini F, Vitali G, Gianaroli L. Spermatogenesis and spectral echo-colour Doppler traces rom the main testicular artery. BJU Int. 2002;90:9038.

33. Tarhan S, Gumus B, Gunduz I, Ayyildiz V, Goktan C. Effect of varicocele on testicular artery blood flow in men: color Doppler investigation. Scand J Urol Nephrol. 2003;37:38-42.

34. Menzies PI. Reproductive health management programs. In: Youngquist RS. Current therapy in large animal theriogenology. Philadelphia: Saunders Elsevier; 1999. p.643-9.

35. Ginther OJ, Garcia MC, Bergfelt DR. Embryonic loss in mares: pregnancy rate, length of interovulatory intervals and progesterone concentrations associated with loss during days 11 to 15 . Theriogenology. $1985 ; 24: 409-17$.

36. Pinggera GM, Mitterberger M, Bartsch G, Strasser H, Gradl J, Aigner F, et al. Assessment of the intratesticular resistive index by colour Doppler Ultrasonography Measurements as a Predictor of Spermatogenesis. BJU Int. 2008;101:722-6.

37. Zelli R, Troisi A, Elad Ngonput A, Cardinali L, Polisca A. Evaluation of testicular artery blood flow by Doppler ultrasonography as a predictor of spermatogenesis in the dog. Res Vet Sci. 2013;95:632-7.

38. Roser JF. Endocrine profiles infertile, subfertile and infertile stallions: testicular reponse to human chorionic gonadotropin in infertile stallions. Biol Reprod. 1995;5:229-39.

39. Bicudo SD, Siqueira JB, Meira C. Patologias do sistema reprodutor de touros. Biologico [Internet]. 2007 [cited 202010 Jan];69:43-8. Available from: http://www.biologico. sp.gov.br/docs/bio/v69_2/p43-48.pdf

40. Filho AAM, Oliveira VK. Torção do testículo: como acontece? [Internet]. ABCMED; 2012 [cited 202014 Mar]. Available from: https://www.abc.med.br/p/saude-dohomem/331755/torcao+do+testiculo+como+acontece.htm

41. Ormond Jk. Recurrent torsion of the spermatic cord. Am J Surg. 1930;12:479-82.

42. Edwards JF. Pathologic conditions of the stallion reproductive tract. Anim Reprod Sci. 2008;107:197-207.

43. Schumacher J. Reproductive system. In: Auer JA, Stick JA. Equine surgery. 4th ed. Missouri: Elsevier Saunders; 2012. p.809-12. 
44. Pavlica P, Barozzi L. Imaging of the acute scrotum. Eur Radiol. 2001;11:220-8.

45. Wallace NG, Amaya M. Normal and developmental variations in the anogenital examination of children. Child Abuse Negl. 2011:69-81.

46. Kryger JV. Acute and chronic scrotal swelling. In: Kliegman R, Lye OS, Bordini BJ, Toth H, Basel D. Nelson Pediatric Symptom-Based Diagnosis. 2nd ed. Philadelphia: Elsivier; 2018. p.330-8.

47. Dudea SM, Ciurea A, Chiorean A, Botar-Jid C. Doppler applications in testicular and scrotal disease. Med Ultrason. 2010;12:43-51.

48. Tanji N, Fujiwara T, Kaji H, Nishio S, Yokokama M. Histologic evaluation of spermatic veins in patients with varicocele. Int J Urol. 1999;7:365-0.

49. Gat Y, Zukerman Z, Bachar GN, Feldberg D, Belenky A, Gornish M. Adolescent varicocele: is it a unilateral disease. Urology. 2003;62:742-7.

50. Gat Y, Zukerman Z, Chakraborty J, Gornish M. Varicocele, hypoxia and male infertility. Fluid mechanics analysis of the impaired testicular venous drainage system. Hum Reprod. 2005;20:2614.

51. Matthews GJ, Matthews ED, Goldstein M. Induction of spermatogenesis and achievement of pregnancy after microsurgical varicocelectomy in men with azoospermia and severe oligoasthenospermia. Fertil Steril. 1998;70:71-5.

52. Gat Y, Bachar GN, Zukerman Z, Gornish M. Varicocele: a bilateral disease. Fertil Steril. 2004;81:424-9.

53. Trojian T, Lishnak TS, Heiman D. Epididymitis and orchitis: an overview. Am Fam Physician. 2009;79:583-7.

54. Wilson KE, Dascanio JJ, Duncan R, Delling U, Lad SM. Orchitis, epididymitis and pampiniform phlebitis in a stallion. Equine Vet Educ. 2007;19:239-43.

55. Jee WH, Choe BY, Byun JY, Shinn KS, Hwan TK. Resistive index of the intrascrotal artery in scrotal inflammatory disease. Acta Radiol. 1997;38:1026-30.

56. Lopez MG, Medina BA, Ortega HR, Rabaza EJ, Romero MMI, Hernandez AMJ. Usefulness of Doppler-color ultrasonography and identification of resistance indexes as early indicators of testicular infarction secondary to orchiepididymitis. Actas Urol Esp. 2000;24:43-7.

57. Brinsko SP. Neoplasia of the male reproductive tract. Vet Clin North Am Equine Pract. 1998;14:517-33.

58. Schumacher J, Varner DD. Neoplasia of the stallion's reproductive tract. In: McKinnon AO, Voss JL. Equine reproduction. Philadelphia: Lea \& Febiger; 1993. p.871-5.

Silva DF, Monteiro GA. Ultrassonografia doppler aplicada ao diagnóstico de distúrbios testiculares em garanhões. Vet. e Zootec. 2020.; 27: 001-017. 
59. Esen B, Yaman MO, Baltac S. Should we rely on Doppler ultrasound for evaluation of testicular solid lesions. World J Urol. 2018;36:1263-6.

60. Bigliardi E, Denti L, De Cesaris V, Bertocchi M, Di Ianni F, Parmigiani E, et al. Color doppler ultrasound imaging of blood flows variations in neoplastic and non-neoplastic testicular lesions in dogs. Reprod Domest Anim. 2018;64:63-71.

Recebido em: 16/04/2020

Aceito em: 15/12/2020 